\title{
Antidiabetic activities of cucurbitane triterpenoids from the fruits of Momordica charantia in streptozotocin-induced diabetic mice
}

\author{
Joo-Hui Han ${ }^{1}$, Kyung-Sun $\mathrm{Heo}^{1}$, Minkyun $\mathrm{Na}^{2}$, Chang-Seon Myung ${ }^{1}$ \\ ${ }^{1}$ Department of Pharmacology, Chungnam National University College of Pharmacy, Korea, ${ }^{2}$ Department of \\ Pharmacognosy, Chungnam National University College of Pharmacy, Korea
}

Improvement of insulin sensitivity can be effective against uncontrolled type 1 as well as type 2 diabetes. Because insulin sensitizers have limitations, there is growing interest in development of new bioactive agents. This study investigated how new cucurbitane-type triterpenoids isolated from the fruits of Momordica charantia affected insulin sensitivity both in vitro and in vivo, and the underlying mechanisms.

Four compounds (C1-C4) isolated from an ethanol extract of M. charantia enhanced glucose uptake in C2C12 myotubes via insulin receptor substrate-1 (IRS-1) and protein kinase B (Akt). The most potent, compound 2 (C2), significantly increased activation of insulin receptor substrate-1 (IRS-1) and downstream signaling pathway, thus subsequent glucose transporter 4 (GLUT4) translocation. Intravenous administration of $\mathrm{C} 2$ to normal and streptozotocin-induced diabetic mice resulted in significant blood glucose-lowering and glycogen-storage effects through the IRS-1 signaling pathway in skeletal muscle but not adipose and liver tissues, indicating that C2 improves hyperglycemia by increasing glucose uptake into skeletal muscle.

These findings suggest the new cucurbitane-type triterpenoids may be useful for the prevention and management of diabetes by improving insulin sensitivity and glucose homeostasis. 